\title{
RINGS WITH INVOLUTION WHOSE SYMMETRIC ELEMENTS ARE CENTRAL
}

\author{
TAW PIN LIM \\ Department of Actuarial Mathematics \\ University of Manitoba \\ Winnipeg, Manitoba R3T 2N2 \\ Canada
}

(Received May 30, 1978 and in revised form February 6, 1979)

ABSTRACT. In a ring $R$ with involution whose symetric elements $S$ are central, the skew-symmetric elements $K$ form a Lie algebra over the commutative ring S. The classification of such rings which are 2-torsion free is equivalent to the classification of Lie algebras $K$ over $S$ equipped with a bilinear form $f$ that is symmetric, invariant and satisfies $[[x, y], z]=f(y, z) x-f(z, x) y$. If $S$ is a field of char $\neq 2, f \neq 0$ and $\operatorname{dim} K>1$ then $K$ is a semisimple Lie algebra if and only if $f$ is nondegenerate. Moreover, the derived algebra $K^{\prime}$ is either the pure quaternions over $S$ or a direct sum of mutually orthogonal abelian Lie ideals of $\mathrm{dim} \leq 2$.

KEY WORDS AND PHRASES. Ring with involution, symmetric and skew-symmetric elements, Lie algebra, symmetric and invariant bilinear form, Cartan's criterion of semisimplicity of lie algebras, pure quaternions, mutually orthogonal abelian Lie ideals.

1980 Mathematics Subject Classification Codes: 16A68, 16A28, 17B05. 
1. INTRODUCTION and MAIN RESULTS.

Let $R$ be a ring with an involution *, 1.e., a map $R \rightarrow R$ such that for all $a, b \in R$

$$
(a+b) *=a *+b *,(a b) *=b * a * \text { and } a * *=a \text {. }
$$

The sets of symmetric and skew-symmetric elements of $R$ are respectively

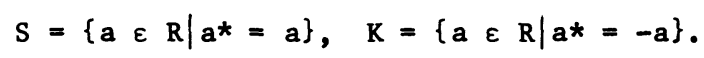

As usual, $[x, y]=x y-y x$ denotes the commutator of $x, y \in R$ and the symbol $z$ denotes the center of $R$.

If the symmetric elements of $R$ are central, i.e., $S \subset Z$, then for abbreviation, $R$ is called a CS-ring.

For all $x \in R, 2 x=x+x^{*}+x-x^{*}$ if $x+x^{*} \varepsilon S, \quad x-x^{*} \varepsilon K$ and thus $2 R \subset S+K$. If $R$ is 2-torsion free then $S \cap K=0$ and hence $\frac{1}{2} \varepsilon R$ Implies that $R$ is a group direct sum $S \oplus K$. If, additionally, $R$ is a CS-ring then for $a \in S, \quad x \in K, a x=x a=-(a x) * \varepsilon K$ and therefore $K$ is a Lie algebra over the commutative ring $S$ with respect to commutation.

We have the following converse:

THEOREM 1. If $S$ is a commutative ring, $K$ is a 2-torsion free lie algebra over $\mathrm{S}$ and $\mathrm{f}: \mathrm{K} X \mathrm{~K} \rightarrow \mathrm{S}$ is an S-bilinear map such that
(1) $\quad f(x, y)=f(y, x)$
(f is symetric)
$f(x,[y, z])=f([x, y], z)$
(f is invariant)

$$
[[x, y], z]=f(y, z) x-f(z, x) y
$$

then the group direct sum $R=S \oplus K$ can be made into a CS-ring by defining the multiplication and the involution, for all $a, b \varepsilon K$, as follows:

$$
(a+x)(b+y)=a b+f(x, y)+a y+b x+[x, y]
$$

$$
(a+x) *=a-x \text {. }
$$

PROOF. Let $a, b, c \in S$ and $x, y, z \in K$. Multiplication in $R$ is associative because 


$$
\begin{aligned}
& ((a+x)(b+y))(c+z)-(a+x)((b+y)(c+z)) \\
= & f(x, y) z+f([x, y], z)+[[x, y], z]-f(y, z) x-f(x,[y, z])-[x,[y, z]] \\
= & f(x, y) z-f(y, z) x-[[z, x], y] \text { by }(2) \text { and Jacobi identity } \\
= & 0 \quad \text { by (3). }
\end{aligned}
$$

From (1), (4) and (5),

$$
((a+x)(b+y)) *=(b+y) *(a+x) * .
$$

Hence, * is an involution in $R$.

Since $K$ is 2-torsion free, $S$ and $K$ are precisely the symmetric and skew-symmetric elements of $R$ and therefore $R$ is a CS-ring.

A CS-ring will, of course, satisfy identities (1) - (3) if we put $f(x, y)=2(x y+y x)$ for all $x, y \in K$.

Note that if $\mathrm{K}$ has an S-basis and $f$ is the dot product then (2) is the triple dot product and (3) is the "triple cross product" with opposite sign, that is, $[[x, y], z]=z X(x X y)$. We must, however, recall that the cross product of vectors is valid only for dimension $\leq 3$ and it can also be ([3,p.61] or [5]) that CS-rings satisfy the standard polynomial of degree 4.

An example of a cS-ring is a ring of quaternions 2 over a 2-torsion free commutative ring $S$, where 2 admits an S-basis $1,1, j, 1 j$ such that given $a, b \in S$

and

$$
i^{2}=a, j^{2}=b, i j=-j i
$$$$
i^{*}=1, i^{*}=-i, j^{*}=-j \text {. }
$$

The skew-symmetric part $\mathrm{K}$ of 2 is a Lie algebra (with respect to commutation) of pure quaternions.

Henceforth, we shall tacitly assume that $\mathrm{K}$ is a Lie algebra over a field $\mathrm{F}$ of char $\neq 2$ and that $K$ is equipped with an F-bilinear form $f$ satisfying identities (1) - (3) such that $R=F \oplus K$ is a CS-ring with the multiplication and the involution defined according to (4) and (5). 
As usual, the derived algebra and the radical of $K$ are respectively $K^{\prime}=[K, K], K^{\perp}=\{x \in K \mid f(x, K)=0\}$. A Lie ideal $I$ of $K$ is a subspace of $K$ with $[I, K] C I$. It can be verified that a Lie ideal of $K$ contained in $K^{\perp}$ Is a proper ideal of $R$.

If $x, y \in K$ then $\langle x, y\rangle$ shall denote the F-subspace of $K$ generated by $x$ and $y$.

PROPOSITION 1. If $\operatorname{dim} K \neq 3$ then $K^{\prime}$ is abelian.

PROOF. We may assume $\mathrm{dIm} K>3$ since the prop. is trivially true for dimension $\langle 3$. From (3), we have $[[x, y], z] \varepsilon\langle x, y\rangle$ for all $x, y, z$ in $k$. Thus, if $x, y, z, w$ are linearly independent vectors of $k$ then

$$
[[x, y],[z, w]] \varepsilon\langle x, y\rangle n\langle z, w\rangle=0 \text {. }
$$

If $w \varepsilon\langle x, y, z\rangle$ where $x, y, z$ are linearly independent then we choose a vector $v$ in $K$ such that $v \notin\langle x, y, z\rangle$ and thus $w+v t\langle x, y, z\rangle$. Consequently, $0=[[x, y],[z, w+v]]=[[x, y],[z, w]]$.

Continuing this argument, we obtain $[[x, y],[z, w]]=0$ for arbitrary $x, y, z, w$ in $K$ and thus $\left[K^{\prime}, K^{\prime}\right]=0$.

PROPOSITION 2. If $f \neq 0$ then $K^{2}$ is an ideal of $R$ contained in $K^{\prime}$ and $\operatorname{dim} K / K^{\prime}=0$ or 1 .

PROOF. If $z \in \mathrm{K}^{\perp}$ then by (2), $f(x,[y, z])=f([x, y], z)=0$ for all $\mathrm{x}, \mathrm{y} \in \mathrm{K}$ and thus $[\mathrm{K}, \mathrm{z}] \subset \mathrm{K}^{\perp}$. Hence, $\mathrm{K}^{\perp}$ is a Lie ideal of $\mathrm{K}$ and an ideal of R. Since $f \neq 0$ there is a nonzero vector $y$ in $K$ with $f(y, y) \neq 0$. If $z \varepsilon K^{\perp}$ then by (3), $[[z, y], y]=f(y, y) z$ and thus $z=f(y, y)^{-1}[[z, y], y] \varepsilon K^{\prime}$. Hence, $\mathrm{K}^{\perp} \subset \mathrm{K}^{\prime}$.

If $\operatorname{dim} K / K^{\prime}>1$ then let $x, y$ be vectors in $K$ which are 1inearly independent modulo $\mathrm{K}^{\prime}$. By (3), $[[\mathrm{x}, \mathrm{y}], \mathrm{K}] \varepsilon\langle\mathrm{x}, \mathrm{y}\rangle \cap \mathrm{K}^{\prime}=0$ which forces $\mathrm{x} \in \mathrm{K}^{\perp}$. Since $\mathrm{K}^{\perp} \subset \mathrm{K}^{\prime}$ we have $\mathrm{x} \in \mathrm{K}^{\prime}$, a contradiction. Hence, $\mathrm{d} d \mathrm{~m} \mathrm{~K} / \mathrm{K}^{\prime}=0$ or 1 . Putting $K^{\prime}=0$, we have COROLLARY 1. If $K$ is abelian and $\operatorname{dim} K>1$ then $f=0$ and $x y=0$ for 
al1 $\mathrm{x}, \mathrm{y} \in \mathrm{K}$.

COROLLARY 2. If $\mathrm{K}^{\prime} \neq 0$ then $\operatorname{dim} \mathrm{K} / \mathrm{K}^{\prime}>1 \Leftrightarrow \mathrm{f}=0 \Leftrightarrow\left[\mathrm{K}^{\prime}, \mathrm{K}\right]=0$.

PROOF. The second equivalence follows from (3). If $f \neq 0$ then by prop. 2 , $\operatorname{dim} K / K^{\prime} \leq 1$

Conversely, if $f=0$ then let $x, y$ be vectors in $k$ with $[x, y] \neq 0$

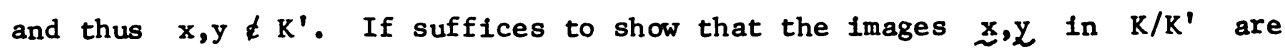
linearly independent over $F$. Indeed, if $y=$ ax for some $a \in F$ then $y-\operatorname{ax} \varepsilon K^{\prime}$ and thus $0=[x, y-a x]=[x, y]$, a contradiction. Hence, $\operatorname{dim} \mathrm{K} / \mathrm{K}^{\prime}>1$

For the Lie algebras that we are considering there is a simple proof of Cartan's criterion for semisimplicity.

THEOREM 2. If $\mathrm{f} \neq 0$ and $\operatorname{dim} \mathrm{K}>1$ then $\mathrm{K}$ has no nonzero abelian Lie ideals if and only if $f$ is nondegenerate.

PROOF. If $\mathrm{K}^{\perp} \neq 0$ then by prop. $2, \mathrm{~K}^{\perp}$ is a nonzero lie ideal contained in $K^{\prime}$. By (3), $\left[K^{\prime}, K^{\perp}\right]=0$ and hence $K^{\perp}$ is abelian.

Conversely, if $\mathrm{K}$ has a nonzero abelian Lie ideal $I$ then let $y, z$ be nonzero vectors in $I$ and $x$ be any vector of $K$ such that $x$ and $y$ are linearly independent. By (3), $f(y, z) x-f(z, x) y=[[x, y], z] \varepsilon[I, I]=0$ and thus $f(y, z)=f(z, x)=0$. Hence, $f(z, k)=0$ and $k^{\perp} \neq 0$.

THEOREM 3. If $f \neq 0$ then $K^{\prime}$ is either a Lle algebra of pure quaternions over $F$ or a direct sum of mutually orthogonal abelian Lie ideals of $K$ with $\operatorname{dim} \leq 2$

PROOF. We may assume $K^{\prime} \neq 0$ for otherwise, prop. 2 would imply that $K$ is of $\operatorname{dim} 1$. We have only to consider the two cases, $\operatorname{dim} K / K^{\prime}=0,1$.

Suppose $K^{\prime}=K$. Since $\left[K^{\prime}, K^{\prime}\right]=[K, K] \neq 0, \operatorname{dim} K=3$ by prop. 1. If $\mathrm{K} \neq 0$ then let $\mathrm{K}=\mathrm{K}^{\perp} \oplus \mathrm{V}$ where $\operatorname{dim} \mathrm{V} \leq 2$ and by (3), $0=\left[\mathrm{K}^{\prime}, \mathrm{K}^{\perp}\right]=\left[\mathrm{K}, \mathrm{K}^{\perp}\right]$ which implies that $K^{\prime}=[\mathrm{V}, \mathrm{V}]$ is of $\operatorname{dim} \leq 1$, contradictory to $K^{\prime}=K$. 
Hence, $K^{\perp}=0$. Since the bilinear form $f$ is symetric and nondegenerate, $K$ has an orthogonal basis $x, y, z$. As $K^{\prime}=K$, the commutators $[x, y],[y, z],[z, x]$ also form a basis of $\mathrm{K}$. By (2), $f(x,[x, y])=f(y,[x, y])=0$ and hence $[x, y]$ is orthogonal to $x$ and $y$. Consequently, $[x, y]=z,[y, z]=a x,[z, x]=b y$ where $a, b \in F$. We can now easily derive from (3) that $f(x, x)=-b, f(y, y)=-a$ and $f(z, z)=-a b$. Hence, $K^{\prime}=K$ is a Lie algebra of pure quaternions over $F$. Suppose $\operatorname{dim} K / K^{\prime}=1$. We have $K^{\perp} \subset K^{\prime}$ by prop. 2. To show $K^{\prime} \subset K^{\perp}$, let $x \in K^{\prime}$ and choose $0 \neq y \notin K^{\prime}$. By (3), $f(y, z) x-f(z, x) y=[[x, y], z] \varepsilon K^{\prime}$ for all $z \in K$ and thus $f(K, x)=0$. Hence, $x \in K^{\perp}$ and $K^{\perp}=K^{\prime}$. Moreover, $0=\left[K^{\prime}, K^{\perp}\right]=\left[K^{\prime}, K^{\prime}\right]$. Since $f \neq 0$, there exists a nonzero vector $e \varepsilon K / K^{\prime}$ with $f(e, e) \neq 0$. Let $d(x)=[x, e]$ for all $x \in K^{\prime}$. By (3), $d^{2}(x)=[[x, e], e]=f(e, e) x$ and hence $d^{2}=f(e, e) I$ where $I$ is the 1dentity map of $K^{\prime}$. Since every nonzero vector $x$ and $K^{\prime}$ is in the d-invariant subspace $L_{x}=\langle x, d(x)\rangle$, it follows from $[2, p .87]$ that $K^{\prime}$ is completely reducible as a module for $d$. Clearly, each $L_{x}$ is an abelian Lie 1deal of $K$ with dim $\leq 2$ and $f\left(L_{x}, L_{y}\right)=0$ for $x \neq y$.

NOTE: This paper constitutes a part of the author's Ph.D. thesis written under the supervision of Professor D. Z. Djokovic at the University of Waterloo, Ontario, Canada.

PRESENT ADDRESS: 491 Parkside Drive Toronto, Ontario CANADA

\section{REFERENCES}

1. Bourbaki, N., Eléments de Mathématique, Groupes et Algebres de Lie, Chap. 1, Hermann, Paris 1971.

2. Curtis C. W. and Reiner, I., Representation Theory of Finite Groups and Associative Algebras, Interscience Pub1ishers, New York 1962.

3. Herstein, I. N., Rings wtih Involution, Univ. of Chicago Press, Chicago 1976. 


\section{REFERENCES}

4. Lim, T. P., Determinants over a class of rings with involution, Ph. D. thesis, Univ. of Water1oo, Canada, 1976.

5. Lim, T. P., Some Classes of Rings with Involution satisfying the Standard Polynomial of Degree 4, submitted to the Pacific Journal of Mathematics. 


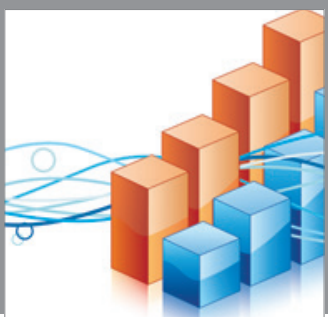

Advances in

Operations Research

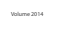

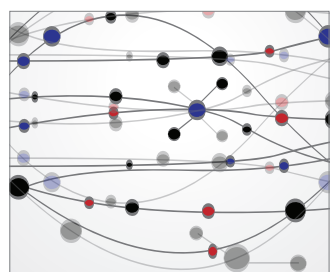

\section{The Scientific} World Journal
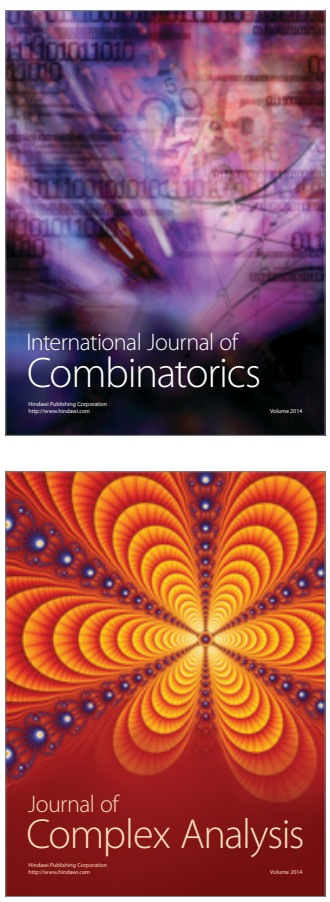

International Journal of

Mathematics and

Mathematical

Sciences
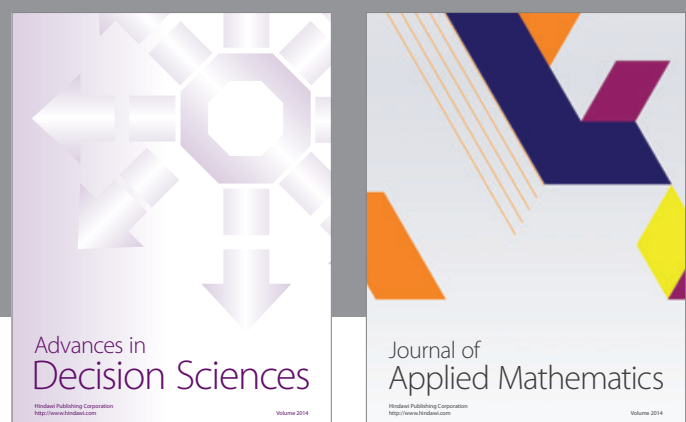

Journal of

Applied Mathematics
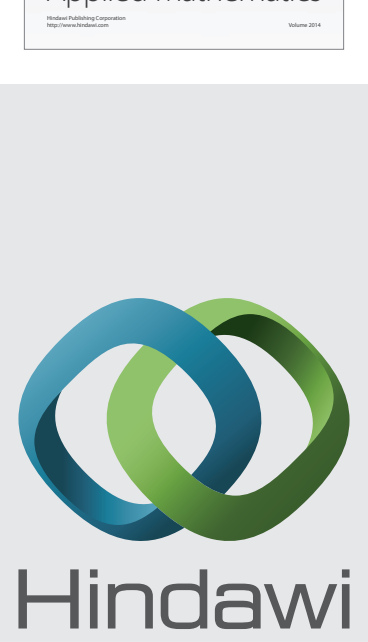

Submit your manuscripts at http://www.hindawi.com
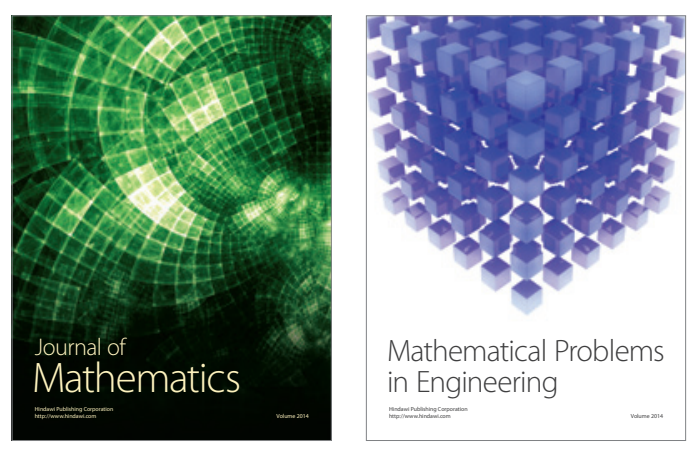

Mathematical Problems in Engineering
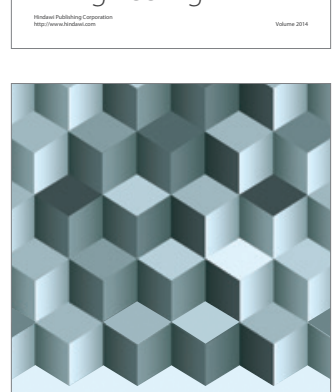

Journal of

Function Spaces
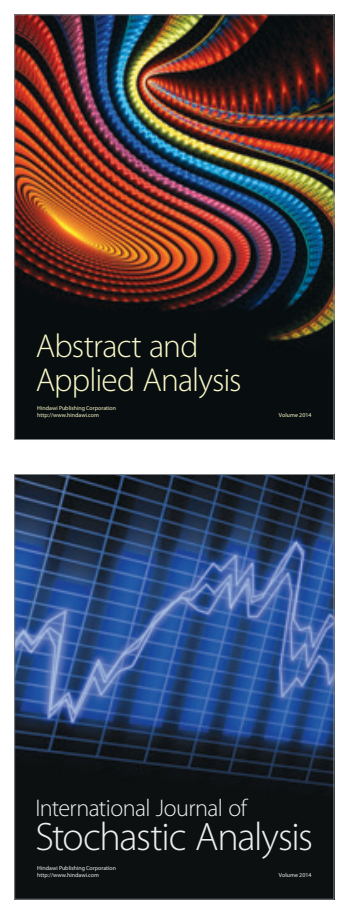

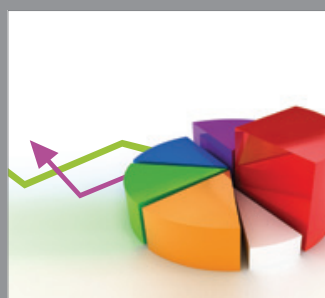

ournal of

Probability and Statistics

Promensencen
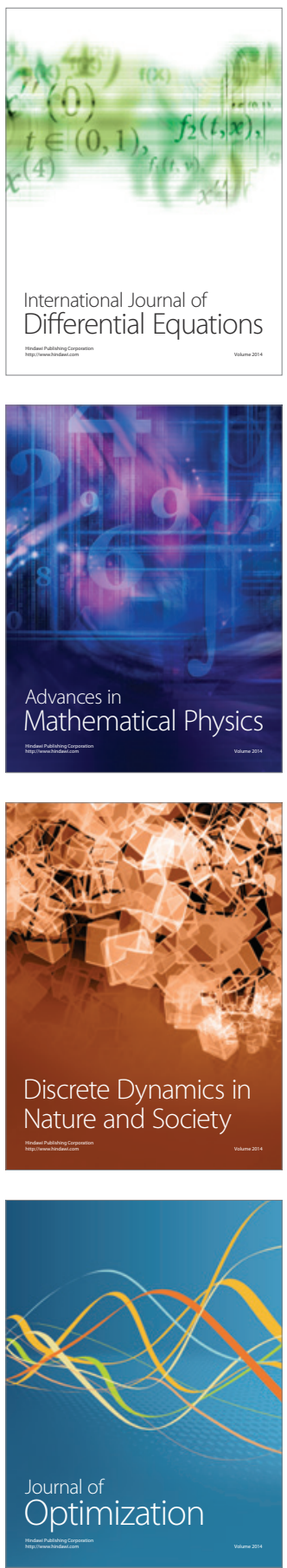